\title{
Applications of SOI-based optical MEMS
}

\author{
Wilfried Noell, Pierre-André Clerc, Laurent Dellmann, Benedikt Guldimann, Hans Peter Herzig, Omar \\ Manzardo, Cornel Marxer, Ken Weible, René Dändliker, and Nico de Rooij
}

\begin{abstract}
After micro-electro mechanical systems (MEMS) devices have been well established, components of higher complexity are now developed. Particularly, the combination with optical components has been very successful and have led to optical MEMS. The technology of choice for us is the silicon-on-insulator (SOI) technology, which has also been successfully used by other groups. The applications presented here give an overview over what is possible with this technology. In particular we demonstrate four completely different devices: a) a $2 \times 2$ optical cross connector (OXC) with an insertion loss of about $0.4 \mathrm{~dB}$ at a switching time of $500 \mu \mathrm{s}$ and its extension to a $4 \times 4$ OXC, b) a variable optical attenuators (VOA), which has an attenuation range of more than $50 \mathrm{~dB}$, c) a Fourier transform spectrometer (FTS) with a spectral resolution of $6 \mathrm{~nm}$ in the visible, and d) an accelerometer with optical readout that achieves a linear dynamic range of $\mathbf{4 0} \mathrm{dB}$ over $\pm 6 \mathrm{~g}$. Except for the FTS, all the applications utilized optical fibers, which are held and selfaligned within the MEMS component by U-grooves and small leaf springs. All devices show high reliability and a very low power consumption.
\end{abstract}

Index Terms-- MOEMS, optical MEMS, optical devices, switches, spectrometers, attenuators, accelerometers.

\section{INTRODUCTION}

$\mathrm{T}^{\mathrm{n}}$ he significant impact of micro-electro mechanical systems (MEMS) in the sensor technology has lead to many new and very small and well controlled devices. In

Wilfried Noell, Pierre-André Clerc, and Nico de Rooij are with University of Neuchâtel, Institute of Microtechnology (IMT), Rue Jaquet Droz 1, CH2007 Neuchâtel, Switzerland, (telephone: +41-32-720-5548, e-mail: Wilfried.Noell@unine.ch).

René Dändliker, Hans Peter Herzig, and Omar Manzardo, are with University of Neuchâtel, Institute of Microtechnology (IMT), Rue Breguet 2, CH-2000 Neuchâtel, Switzerland, (e-mail: hanspeter.herzig@unine.ch).

Laurent Dellmann and Benedikt Guldimann were with University of Neuchâtel, IMT, CH-2007 Neuchâtel, Switzerland and are now with University of California, Cory Hall, Berkeley, CA 94720, USA (e-mail: dellmann@eecs.berkeley.edu and benedikt@eecs.berkeley.edu).

Cornel Marxer was with University of Neuchâtel, IMT, CH-2007 Neuchâtel, Switzerland and is now with Sercalo Microtechnology Ltd., Landstrasse 151, FL-9494 Schaan, Principality of Liechtenstein (telephone: +423-237-5797, e-mail: cornel.marxer@sercalo.com). Sercalo Microtechnology Ltd. is a start-up company, that commercializes optical MEMS developments for telecommunication applications.

Ken Weible is with Weible OpTech, Rue Jaquet Droz 7, CH-2007 Neuchâtel, Switzerland (e-meil: weible@suss.ch).

The bi-stable 2x2 OXC, which is described in Chap. III.A. and in Fig. 2b, was fabricated by the University of Neuchâtel (IMT), Switzerland, under contract of OCLI, Inc./JDS Uniphase. It was first published as a postdeadline contribution by OCLI on the optical MEMS conference in Kauai, Hawaii, USA, in August 2000.

Financial support for part of the work was provided by the Swiss Federal Office for Education and Science (Collaborative Projects of the European Union). the optical domain MEMS components are becoming more and more relevant, particularly for the telecommunication industry and optical sensor technology.

Regarding the telecommunication industry, there are four main device domains: Amplifiers, switches or optical cross connectors (OXC), filters and variable optical attenuators (VOA). The amplifiers are made nowadays by optically pumped Erbium doped fibers and are usually referred to as EDFA (Erbium Doped Fiber Amplifiers). These purely optically working devices are fast and do not require any mechanical components. This becomes very different, when looking at the other three domains. OXC and VOA are based on mechanical elements and are hence relatively slowly working devices. This is accepted, because these devices have to work in routers and amplifiers and under conditions that are almost static compared to the modulation frequencies of typically several $\mathrm{GHz}$ of the optical signals. Filters for wavelength division multiplexing (WDM) are based on tunable thin film devices or integrated optical elements, which are tuned either mechanically or thermally, and are hence also relatively slow. Two different optical MEMS components that are relevant for telecommunication applications are presented in this paper: $\mathrm{A} 2 \times 2$ and $4 \times 4$ OXC and an attenuator.

Although many optical MEMS devices are driven by the telecommunication industry other research and industry areas are relevant as well. Optical spectroscopy is one of this fields. When high spectroscopic resolution is required, optical spectrometers are still relatively big. The same applies to infrared spectroscopes where almost no small solutions are available. In this branch optical MEMS can lead to important technological breakthroughs. A step towards this optical domain is a MEMS based Fourier transform spectrometer (FTS), which will be presented here.

Since it is very easy to measure optical intensities, the modulation of light by a MEMS component can be used as an effective measurement signal of a mechanical movement or displacements. Utilizing the light modulation of a MEMS based fiber coupler lead to an highly accurate accelerometer, the last device presented in this document.

This publication delivers a brief overview over different optical MEMS activities of IMT that are based on siliconon-insulator (SOI) technology. It would be beyond the scope of this paper to describe all effects, particularities, and the in-depth theoretical background of the presented 
devices. The reader may refer to the corresponding previously published and specialized publications which are listed in the references.

The authors focus on the activities of IMT. There are many other groups that have been working very successfully on similar devices based on SOI technology. E.g. the group around O. Solgaard (Stanford University, CA, U.S.A.) work actively on scanning mirrors, light modulators and other optical MEMS's. Ming Wu and Norman Tien (Cornell University, University of California of Los Angeles and Davis, respectively, U.S.A.) as well as Hiroshi Toshiyoshi and Hiroyuki Fujita (University of Tokyo, Japan) have shown many novel devices based on SOI surface micromaching. The department of Youn Tae Kim (Electronics and Telecommunications Research Institute (ETRI), Daejeon, Korea) works successfully on switchable mirrors in collaboration with Masayoshi Esashi (Tohoku University, Japan). Optical MEMS activities based on SOI technology are also pursued by the group of Young-Ho Cho (KAIST, Taejon, Korea). Many companies, e.g. JDS Uniphase, Lucent Technologies, Kionix, Tronics, OMM, Leti, Texas Instruments and many more utilize SOI processes for their products as well.

A particularity of the IMT silicon bulk micromaching lays in the used substrate: Handle and device wafer are both made of single crystalline silicon, which is different from the similar looking surface micromaching which utilizes polycrystalline silicon on top of the buried $\mathrm{SiO}_{2}$ layer. The single crystal silicon delivers smoother surfaces after the dry etching and has in general better mechanical properties. All the optical MEMS components that we demonstrate here are based on one and the same technology, the SOI technology, whose basics will be briefly explained in the following paragraph.

\section{SOI TECHNOLOGY}

The big advantage of the SOI technology is its simplicity and small number of process steps, because there is usually only one patterning and one depositing step involved. The most critical process step, however, is the deep reactive ion etching (DRIE) that determines the structure itself and surface roughness of the sidewalls of the crucial optical elements of the device (Fig. 1).

The substrate for this process is a composite wafer, whose substrate is a silicon wafer of $100 \mathrm{~mm}$ in diameter. Above the substrate is a silicondioxide layer of $2 \mu \mathrm{m}$ thickness. The top most layer is the so called silicon device layer, which has a thickness of $75 \mu \mathrm{m}$ in our case (Fig. 1a).

The first step is the photolithography that patterns the positive photoresist (Fig. 1b). The photoresist is used to dry-etch the silicon of the device layer by deep reactive ion etching (DRIE) (Fig. 1c). The DRIE etched trenches comprise vertical walls. This way, rather narrow structures a)

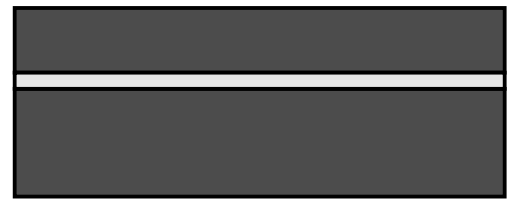

silicon

$\mathrm{SiO}_{2}$

silicon

b)

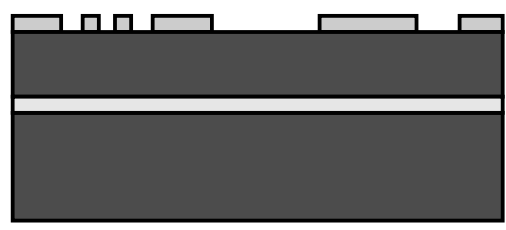

photo resist

c)

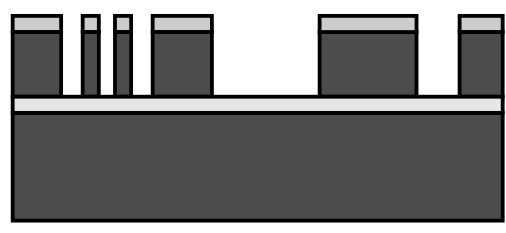

DRIE trenches

d)

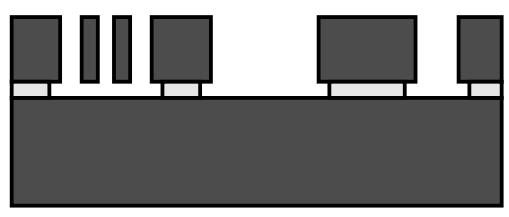

HF etch

e)

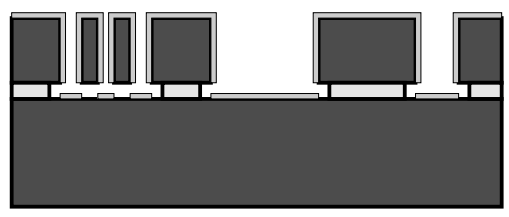

metallisation

f)

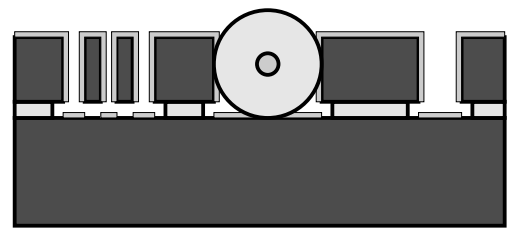

assembly

Fig. 1. SOI technology: a) The process starts with an SOI wafer which is a composite of a silicon substrate wafer, a buried silicondioxid (BOX) layer, and a device layer, which is in our case $75 \mu \mathrm{m}$ high. b) and c) The wafer is patterned by DRIE utilizing a positive photoresist mask. d) The structures are released by wet-etching the BOX underneath the structures that have to move freely during the actuation. e) The metallisation improves the surface conductivity and reflectivity. The structures that are not connected via the patterned silicon are electrically isolated from each other by BOX pedestals. f) Integrated alignment structures such as U-grooves are used for the assembly of optical components, e.g. optical fibers.

of about 2 to $5 \mu \mathrm{m}$ can be achieved with very smooth sidewalls, which is not possible with wet etching solutions such as $\mathrm{KOH}$. The buried $\mathrm{SiO}_{2}$ serves as an etch stop for the DRIE. Since the etch rate is different in the middle of the wafer and at its outer regions as well as for narrow and wide structures, an etch stop is absolute necessary to guarantee that all structures have the very same height and width required by the mask design. After the DRIE the resist is removed by oxygen plasma etching.

To release the structures the buried $\mathrm{SiO}_{2}$ has to be 

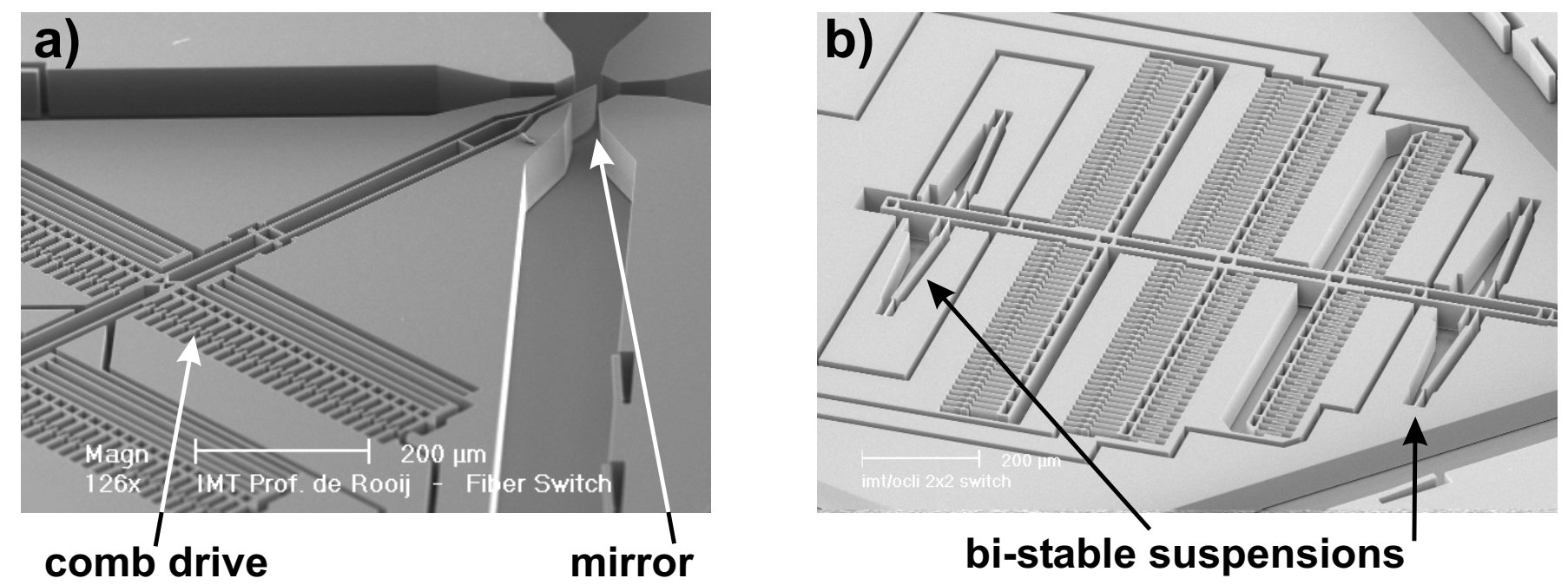

Fig. 2. a) The $2 \times 2$ switch can hold four optical fibers. The thin mirror is actuated by comb drive actuators. Leaf springs create the counter force and the suspension of the beam that holds the mirror. b) In a further design, the suspensions are bi-stable. In this design the switching is performed by voltage pulses. In both devices the optical fibers are held by small leaf springs which are integrated in the sidewalls of the U-grooves.

removed (Fig. 1d). The etching takes place in 50\% concentrated hydrofluoric acid (HF). The HF etch time is adjusted such, that those parts are completely underetched, which have to move freely during the later actuation. $\mathrm{SiO}_{2}$ pedestals, however, have to remain under the supporting parts of the MEMS structure. The $\mathrm{SiO}_{2}$ also serves as an insulator between the different electrodes of the final device.

To increase the conductivity of the device surface and for a proper reflectivity of the mirrors, gold is deposited by electron beam evaporation over the whole device without any masking or patterning (Fig. 1e). In the final step different optical components, e.g. optical fibers, can be attached to the MEMS component (Fig. 1f). Guiding structures, e.g. U-grooves, are integrated in the MEMS design to guarantee a high alignment among the different optical components. The U-grooves can comprise little leaf springs to hold the optical components in place.

\section{DEVICES FOR TELECOMMUNICATION}

There are two devices that have proven successful when entering into applications for fiber optic communication: a) A 2x2 optical fiber switch and b) a variable optical attenuator (VOA) [1,2]. An extension of the $2 \times 2$ switch towards a $4 \times 4$ switch of higher complexity will also be presented.

\section{A. $2 \times 2$ Optical Fiber Switch}

The $2 \times 2$ OXC has two input and two output fibers. In the MEMS component, the four fibers sit in $75 \mu \mathrm{m}$ high U-grooves and are oriented at right angles to each other. Their endfaces all meet at one point, where a movable gold-coated mirror is located. This way, the light of either one of the input fibers can be switched to either one of the output fibers.

To enhance the optical performance and minimize the loss, all the optical components are completely surrounded by refractive index matching fluid. The device is placed into a hermetically sealed housing to prevent leaking of the oil. The index matching fluid reduces two effects: first the index step between the fiber endfaces and the ambient and secondly the divergence of the freely propagating light in the ambient and hence the beam diameter, which both leads to an increasing coupling efficiency between the fibers.[1]

The mirror is attached to a long beam that is electrostatically actuated by comb drives (Fig. 2a). In the first design suspending leaf springs created the restoring mechanical force. The driving voltage to overcome the linearly acting mechanical counter force is in the order of 50 to $100 \mathrm{~V}$. Applying no voltage resets the switch back into its zero or ground position.

In a further more advanced design, the switch is bistable or more mechanically spoken latching (Fig. 2b). Every time the switch needs go from one to the other of its two possible positions only a short voltage pulse needs to be applied. The mirror then remains in the corresponding position without any driving voltage.[3]

The optical and mechanical properties of the switch are excellent. The insertion loss is between 0.3 and $0.5 \mathrm{~dB}$. The switching time is about $500 \mu$ s and the live time was tested to be $5 \cdot 10^{9}$ cycles at $85^{\circ} \mathrm{C}$ (corresponds to $2 \cdot 10^{11}$ at room temperature). The cross talk between the fibers is as low as $-70 \mathrm{~dB}$. There is basically no polarization dependency to the coupling efficiency. 


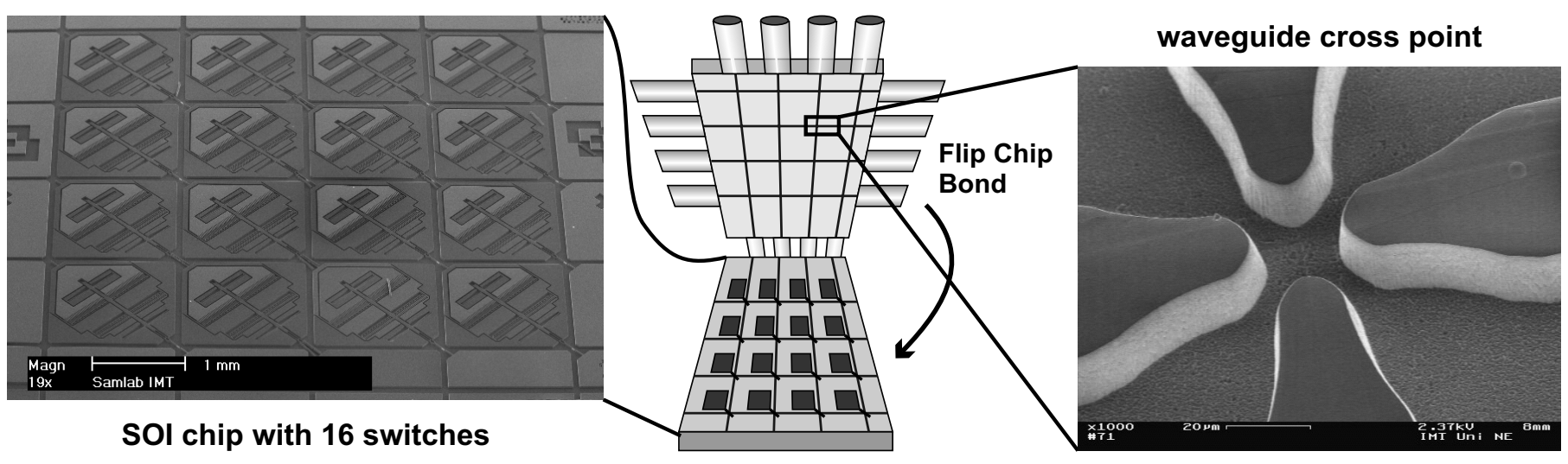

Fig. 3. In the new concept of the $4 \times 4$ OXC, the scalability of the $2 \times 2$ OXC is tested. The design includes two chips. The bottom chip holds 16 electrostatically actuated mirrors. The switches are bi-stable trough a latching mechanism. The top chip includes the integrated optical waveguides and the electronic wiring for the bottom chip. Attached to the waveguide chip are the optical fibers. In order to insert the waveguides into the chip structure in the same fashion as the optical fibers into the $2 \times 2$ OXC, the waveguides are etched by DRIE. The two chips are to be connected by flip-chip bonding.

\section{B. $4 x 4$ Switch Design}

The $2 \times 2$ switch has demonstrated high reliability. Thus our current development examines the scalability of the technology to achieve NxN OXC [4]. The first approach aims at a $4 \times 4$ OXC and consists of $162 \times 2$ MEMS switches that will be optically interconnected by integrated optical waveguides (Fig. 3). The input and output connections will be 16 standard single mode optical fibers. The $4 \times 4$ OXC essentially consists of two components, a bottom chip, which contains the 16 bi-stable silicon switches, and a top chip holding the waveguides and the electrical connections for the switches. The two chips will be combined by standard flip chip bonding technology. The particular challenge in this projects are a) the deep reactive ion etching (DRIE) of the integrated optical waveguides so they can be inserted into the switches in the same fashion as in the $2 \times 2$ fiber switch and b) the assembly of the complete system.

The weakly guiding and strip-loaded integrated optical waveguides are fabricated on a silicon wafer with $8 \mu \mathrm{m}$ thermally grown $\mathrm{SiO}_{2}$ [5]. The buffer layer, the striploaded core layer as well as the cladding layer are deposited by plasma enhanced chemical vapor deposition (PECVD). The buffer layer consists of $3 \mu \mathrm{m} \mathrm{SiO}_{2}$. The strip layer and core ridge are made of $\mathrm{SiO}_{\mathrm{x}} \mathrm{N}_{\mathrm{y}}$ by depositing a $350 \mathrm{~nm}$ thick layer, which is thinned down next to the 6 $\mu \mathrm{m}$ wide waveguide core to $100 \mathrm{~nm}$ by reactive ion etching (RIE). In the last step a $\mathrm{SiO}_{2}$ cladding layer of $15 \mu \mathrm{m}$ thickness is PECVD deposited on top of the $\mathrm{SiO}_{\mathrm{x}} \mathrm{N}_{\mathrm{y}}$ structure. The specific loss of the integrated optical waveguides can be as low as $0.2 \mathrm{~dB} / \mathrm{cm}$ at $\lambda_{0}=1300 \mathrm{~nm}$ and $0.4 \mathrm{~dB} / \mathrm{cm}$ at $\lambda_{0}=1550 \mathrm{~nm}$.

Due to the thin core ridge, the waveguide is rather weakly guiding perpendicular to the layer plane. Thus the beam shape is expanded in this direction, which leads to an overall relatively round beam shape and a highly improved coupling efficiency between the integrated waveguide and the attached fiber.

To connect the integrated optically waveguides with optical fibers, the waveguide wafers need smooth endfaces. This can be achieved by either a special polishing dicing technology or standard dicing and additional polishing with diamond powder. This way fiber-to-waveguide coupling loss can be as low as $0.35 \mathrm{~dB}$ per facet.

In order to insert the integrated optical waveguides into the U-grooves of the switches and in between the mirrors, the waveguides need to be pattern by DRIE [6]. After performing etched tests on quartz glass wafers with an SU8 photoresist mask [7,8], the silica-on-silicon wafers with the waveguides were patterned identically (Fig. 3, right image). The surface roughness is still to be optimized but can be smoothed out optically to a certain degree by indexmatching oil.

\section{Variable Optical Attenuator (VOA)}

When optical signals are traveling in fibers for several kilometers their intensity becomes less due to absorption, scattering and bending losses in the fiber network. When the signal intensity falls under a certain threshold, the signal is amplified by EDFA. The amplification, however, is wavelength dependent. Therefore, the channels are split after the amplification by WDM and the different intensities of each channel are equalized to the same value. Instead of additional amplification of each channel, the intensities are attenuated to the required value by a VOA. After the attenuation the channels are combined again by a wavelength multiplexer (WM). Since the amplification is relatively constant, the VOA has to be set to a certain attenuation value where it remains statically until readjustments have to be done due to losses in the WDM or a change in the EDFA.

The VOA presented here is based on the same MEMS 

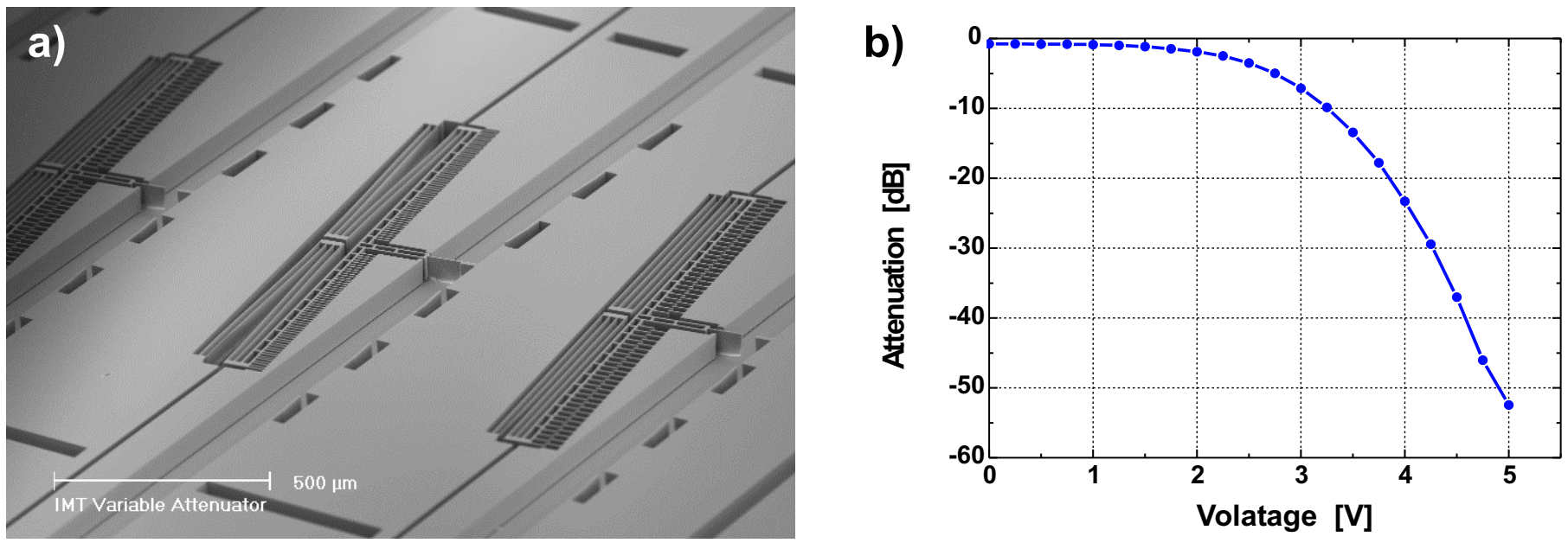

Fig. 4. a) The variable optical attenuator (VOA) moves a shutter in between two fibers. B) The attenuation of the VOA has a dynamic range of over $50 \mathrm{~dB}$.

concept as the switch described above: A mirror that is rotated by $82^{\circ}$ to the axis of light propagation can move linearly between two opposing fibers that are held by small leaf springs in two U-grooves (Fig. 4a).[2] The slight tilt prevents the back reflection into the input fiber. The attenuation efficiency versus the driving voltage is depicted in Fig. 4b.

The attenuation is not linear with the driving voltage and thus the VOA has to be calibrated prior to its use in a power management setup.

Since the footprint of the MEMS VOA is very small, several VOA's are fabricated on one chip very close to each other.

\section{FOURIER TRANSFORM SPECTROMETER (FTS)}

Fourier transform (FT) spectroscopy is a well-known technique to measure the spectra of a weak and extended light source whereas it offers a higher signal-to-noise ratio than other methods [9]. Commonly used FT spectrometers require a mirror scanning mechanism with very high precision, resulting in a large device size at high costs. Low-cost miniature spectrometers, however, are key components that permit the fabrication of small-size, portable sensor solutions for applications such as photometers in quality management and spectrometers in analysis tools.

The most common way of fabricating an FTS is utilizing a Michelson interferometer configuration with scanning mirrors. The output signal of the interferometer is the variation of the light intensity $I_{R}$ as a function of the optical path length $\delta$. The relation of $I_{R}$ to $\delta$ is referred to as the interferogram. The wavelength power spectrum and the recorded interferogram $I_{R}(\delta)$ are related by a Fourier transformation. Therefore, a spectrum of a light source can be obtained by simply recording $I_{R}(\delta)$ and a subsequent FT.

Most microfabricated FTS utilized a tilted mirror and a photodiode array in a stationary Michelson interferometer configuration [10]. Such set-ups, however, offer only a rather poor resolution. Therefore, a new fabrication concept was pursued that includes a compact MEMS scanning mirror with a small footprint of only $5 \times 4 \mathrm{~mm}^{2} .[11]$ The schematics of this Michelson
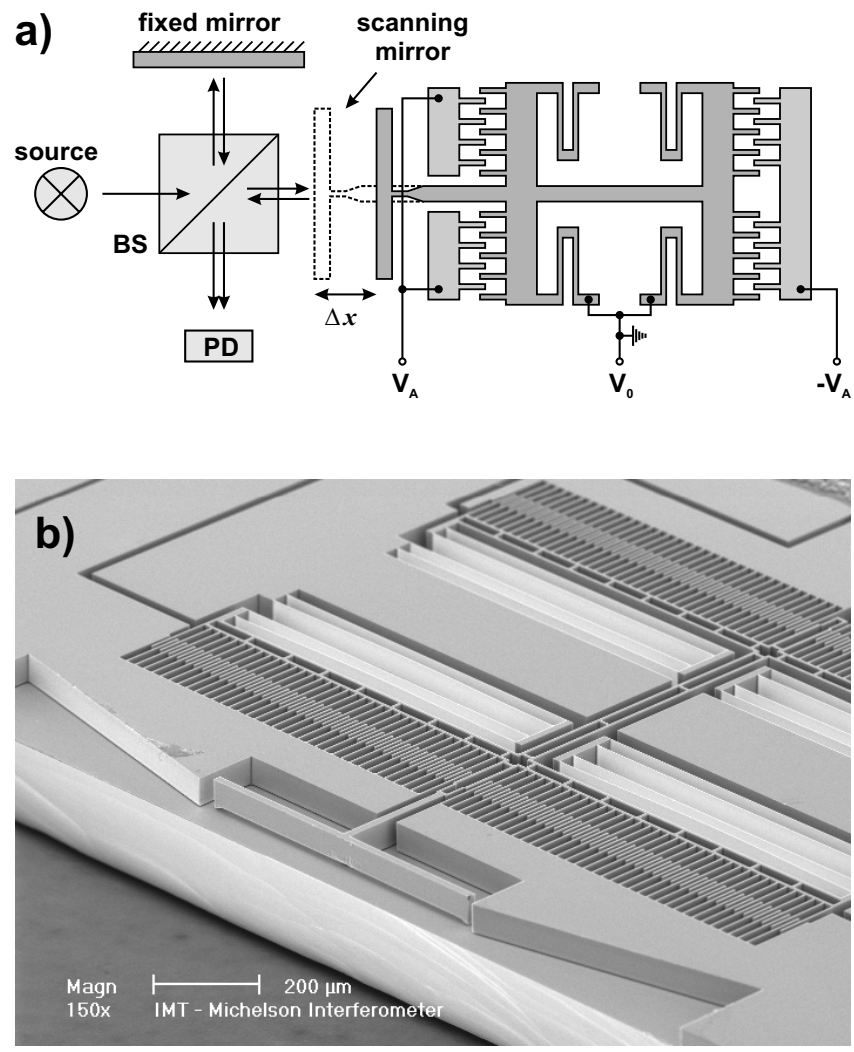

Fig. 5. a) Concept of Fourier transform spectrometer (FTS). In the real setup, the beam splitter (bs) and the fixed mirror are macroscopic. The movable mirror was driven by a push-pull configuration. b) The MEMS mirror is much larger than in the OXC and the VOA to increase the reflecting area. 
interferometer set-up is depicted in Fig. 5a. The light from the source is divided by a standard macroscopic beam splitter to the movable MEMS mirror and the photodetector. The other mirror opposing the photodetector (PD) is fixed. The design of the MEMS mirror is derived from the $2 \times 2$ switch structure by rotating the mirror by $90^{\circ}$ in respect to the actuation direction (Fig. 5b). The mirror size was enlarged to increase the reflecting surface.

In order to achieve the obligatory linear relation between the driving voltage and the mirror displacement, a push-pull type driving voltage configuration was chosen by setting the inner combs to one and the same electrical potential $V_{0}$ and the other two combs on opposing voltages $V_{A}$ and $-V_{A}$, respectively. This way $\Delta x$ is proportional to $V_{A}$, when at the same time $V_{0}$ is kept constant.

To test the set-up a He-Ne laser was focused onto the two mirrors via the beam splitter. The driving voltage for the movable mirror was either $V_{A}= \pm 5 \mathrm{~V}$ for half the travel distance or $V_{A}= \pm 10 \mathrm{~V}$ for the maximum achievable travel distance of $\Delta x=38.5 \mu \mathrm{m}$ which corresponds to an optical path difference of $\delta=77 \mu \mathrm{m}$. While the small voltage range yielded an almost linear mirror displacement response, the full range showed a slight non-linearity as well as a small hysteresis. Both effects, however, remained almost constant for several cycles and could be eliminated by calibrating the voltage response of the mirror displacement. After this interferometric calibration, the linewidth of the He-Ne laser was measured to be about $6 \mathrm{~nm}$, whereas the peak position was repeatedly accurate to about $1 \mathrm{~nm}$. Since the real linewidth of the laser is only a few picometers, the measured linewidth corresponds to the actual resolution of the MEMS FTS.[11]
A new design with integrated collimators, fixed mirrors and guiding structures for a sheet-type beam splitter as well as a tunable laminar grating are currently under investigation.[12]

\section{ACCELEROMETER}

Accelerometers are needed in several technical areas where the changing speed of an object has to trigger an important action or simply needs to be monitored. The probably most famous application for an accelerometer is the airbag activator in vehicles. Other more general applications are quantitative vibration measurements for heavy machines. The change of a direction of a moving object induces a change in its angular velocity, which can be measured with an accelerometer.

Although there are many accelerometers that are based on piezoelectric ceramics or the electrical detection of a moving mass, silicon MEMS based accelerometers with optical readout become more and more sensitive, reliable, and important.[13,14,15,16]

The accelerometer presented here is based on the measurement of an optical modulation caused by a moving mass (Fig. 6a). Using purely optical signals has the big advantage, that the device performance is immune to electromagnetic interferences (EMI).[17] The optical source is an LED. The optical detection utilizes the partial and simultaneous light coupling from one source fiber into two output fibers. The fibers are held and self-aligned by integrated U-grooves in the same fashion as in the OXC and VOA described above (Fig. 6b). The light of the source fibers hits the point of a $\mathrm{V}$-shaped mirror, which splits and couples the light evenly into the two identical output
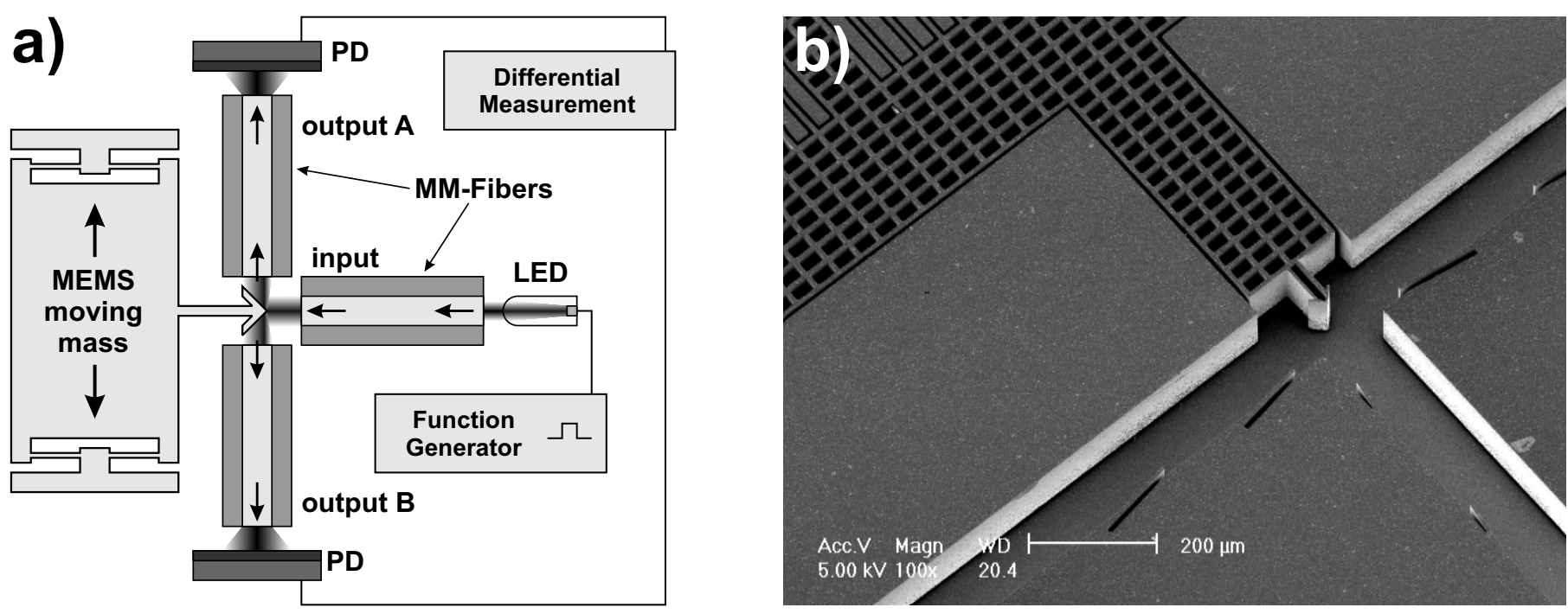

Fig. 6. a) In the optical MEMS accelerometer the light from the input fiber is split by a V-shaped mirror simultaneously into the two output fibers A and B. The signal from the photodectors are subtracted from each other to achieve a differential measurement. The dynamic range of the usable and linear response of the moving mass is about $40 \mathrm{~dB}$ for $\pm 6 \mathrm{~g}$ at a bandwidth of $300 \mathrm{~Hz}$. b) The fibers are inserted into the MEMS component via U-grooves and held by small leaf springs. The accelerometer is damped by a squeezed air film which is in a small gap in between the moving mass and the surrounding structure, which is visible in the upper left corner. 
multimode fibers. The mirror is attached to a movable mass, whose moving is damped during its displacement by squeezing an air film, which is located in between the mass and its surrounding silicon structure and which can be seen in the upper left corner of Fig. 6b.[18]

The output fibers guide the light to two photodiodes. This arrangement allows a differential measurement of the optical power. To obtain a sufficiently linear response, however, only tiny displacements around the zero position are allowed. Therefore, the device is designed to have a maximum displacement of $\pm 0.8 \mu \mathrm{m}$, which is a tenth of the damping air gap width and only a small fraction of the displacement necessary to achieve the maximum possible light modulation. Since the total light modulation corresponds to an electronic signal-to-noise level of $102 \mathrm{~dB}$ at $300 \mathrm{~Hz}$, the mechanical limitations only allow to use an effective dynamic range of about $40 \mathrm{~dB}$ or a measurable displacement of about $0.6 \AA$. Thermal-mechanical noise is negligible. In spite of this limitations the performance is comparable to more sophisticated and more expensive Fabry-Perot based devices of Ref. 16 and it outperforms previously published opto-mechanical MEMS accelerometers.

\section{CONCLUSION}

Optical MEMS components based on the relatively simple SOI technology offer several advantages over standard components. They deliver a high reliability, need only a small footprint of a few square millimeters, integrate alignment structures for small optical components, and show negligible power consumption when driven with electrostatic actuators. This means, that optical MEMS components offer the opportunity to create small, comprehensive, and highly sophisticated systems of a high level integration. Since the DRIE etch depth is not limited to the presented device layer heights of 75 and $100 \mu \mathrm{m}$, other heights can be utilized as well when it is required by the application. More sophisticated MEMS designs with a higher level of integration are conceivable, although more functions complicate the device fabrication and can lead to a lower process or trade-off in the functionality of the single components.

\section{ACKNOWLEDGMENT}

The authors like to thank the staff members of their corresponding groups for useful help in finding out useful details in the process development as well as in the microassembly. Particularly, we are very grateful to Martin Hoffmann from the University of Dortmund (Germany) for providing the integrated optical waveguide wafers.

\section{REFERENCES}

[1] C. Marxer and N.F. de Rooij, "Micro-optomechanical 2 × 2 switch for single-mode fibers based on a plasma-etched silicon mirror and electrostatic actuation", Journal of Lightwave Technology, Vol. 17, No. 1, pp. $2-6,1999$.

[2] J.H. Lee, M.L. Lee, W.I. Jang, C.A. Choi, and Y.T. Kim, "A planar latch-up microactuator driven by thermoelastic force", Micromachined Devices and Components VI, Eric Peeters, Oliver Paul, Editors, Proc. of SPIE, Vol. 4176, 2000.

[3] C. Marxer, P. Griss, N.F. de Rooij, "A variable optical attenuator based on silicon micromechanics", IEEE Photonics Technology Letters, Vol. 11, no. 2, pp. 233-235, 1999.

[4] L. Dellmann, W. Noell, C. Marxer, K. Weible, M. Hoffmann and N.F. de Rooij, "4x4 Matrix Switch Based On Mems Switches And Integrated Waveguides", Transducers '01 Eurosensors XV, The 11th International Conference on Solid-State Sensors and Actuators, Munich, Germany, June 10-14, pp. 1332-1335, 2001.

[5] M. Hoffmann, P. Kopka and Edgar Voges, "Lowloss fiber-matched low-temperature PECVD waveguides with small-core dimensions for optical communication systems", IEEE Photonics Tech. Letters, Vol. 9, No. 9, pp 1238-40, 1997.

[6] R. Völkel, P. Nussbaum, J.-Ch. Roulet, P. Blattner, K. J. Weible, H. P. Herzig, "Design, fabrication and testing of micro-optical components for sensors and microsystems", SPIE 3099, pp. 196-211, 1997.

[7] L. Dellmann, S. Roth, C. Beuret, G.-A. Racine, H. Lorenz, M. Depont, P. Renaud, P. Vettiger and N.F. de Rooij, "Fabrication process of high aspect ratio elastic and SU-8 structures for piezoelectric motor applications", Sensors and Actuators, A Physical, vol. 70, pp. 42-47, 1998.

[8] K. Y. Lee, N. LaBioanca, S.A. Rishton, S. Zolgharnain, J.D. Gelorme, J. Shaw and T.H.P. Chang, "Micromachining Applications of a high resolution ultrathick photoresist", J. Vac. Sci. Technol. B 13, pp. 3012 3016, 1995.

[9] R. J. Bell, "Introductory Fourier Transform Spectroscopy", Academic, New York, 1972.

[10] O. Manzardo, P. Kipfer, H. P. Herzig, H. Teichmann, M. T. Gale, and P. Ehbets, "Compact Fourier transform spectrometer for applications requiring moderate resolution", in Optical Spectral Analysis Microsystem, Engelberg, EOS Topical Meetings Digest Series, Vol. 16, pp. 52-53, 1998

[11] O. Manzardo, H.-P. Herzig, C.R. Marxer, N.F. de Rooij, "Miniaturized time-scanning Fourier Transform Spectrometer based on silicon technology", Opt. Lett., Vol 24, 1705-1707, 1999

[12] O. Manzardo, B. Guldimann, C. Marxer, N.F. de Rooij, H.-P. Herzig, "Optics and Actuators for Miniaturized Spectrometers", Optical MEMS 2000, Kauai, August 21-24, 2000.

[13] L. Jonsson and B. Hök, "Multimode fiber-optic accelerometers", SPIE 514 1984, pp. 191-194, 1984.

[14] D. Uttamchandani, D. Liang and B. Culshaw, "A micromachined silicon accelerometer with fiber optic interrogation”, SPIE 1792, pp. 27-33, 1992.

[15] J. Marty et al., "Fiber-Optic accelerometer using silicon micromachined techniques", Sensors and Actuators A, Vol 46-47, pp. 470-473, 1995.

[16] G. Schröpfer, et al., "Lateral optical accelerometer micromachined in (100) silicon with remote readout based on coherence modulation", Sensors and Actuators A, Vol. 68, pp. 344-349, 1998.

[17] A. J. Jacobs-Cook, "MEMS versus MOMS from a systems point of view", J. Micromech. Microeng., Vol. 6, pp. 148-156, 1996.

[18] B. Guldimann, P. Dubois, P.-A. Clerc, N.F. de Rooij, "Fiber Optic MEMS Accelerometer with high mass displacement Resolution", Transducers '01 Eurosensors XV, The 11th International Conference on Solid-State Sensors and Actuators, Munich, Germany, June 10 $14,2001$. 
Wilfried Noell is with the Institute of Microtechnology (IMT) of the University of Neuchâtel, Switzerland, since 1998. After working for more than two years on tools for nanoscience he became responsible for the group's activities on optical microsystems in the beginning of 2001. He received his Ph.D. from the physics department of University of Ulm, Germany, in 1998. The Ph.D. work was pursued externally at the Institute for Microtechnology Mainz (IMM), Germany, on the development of a microfabricated optical near-field sensor. He finished his physics studies with a diploma degree at the Technical University of Darmstadt (TUD), Germany, in 1994. His diploma thesis was a joined work between the applied optics group of the physics department of TUD and the research and technology center (FTZ) of the Deutsche Telekom on integrated optical waveguides based on InP.

Laurent Dellmann received a degree in Microtechnology from the Swiss Federal Institute of Technology of Lausanne (EPFL), Switzerland in 1996. He obtained his Ph.D. from the Institute of Microtechnology (IMT) at the University of Neuchatel in Switzerland, in 2000 with a dissertation on microfabrication of piezoelectric motors for a wristwatch application. After a postdoctoral at the Institute of Microtechnology of the University of Neuchatel in Switzerland in 2000, where he worked on the development of integrated optical switch matrix for telecommunication, he joined the Bio-engineering department at the University of California at Berkeley as a visiting post-doc in 2001, where he is involved in the development of BioMEMS-type devices.

Benedikt Guldimann currently works as a postdoc at the University of California in Berkeley on optical microsystems. He received his Ph.D. on optical microsensors, actuators and microfluidic systems from the University of Neuchâtel in 2001, where he worked since 1996 at the Institute of Microtechnology (IMT). Prior to his Ph.D. program he worked at the Swiss Federal Institute of Technology of Lausanne (EPFL), Switzerland, as an assistant for fiber optics. In 1995 he joined the ESAESTEC Instrument Technology Division, Noordwijk, The Netherlands, as a young graduate traineee on microoptics and metrology. Between 1993, when he received his diploma in electronics physics from the University of Neuchâtel, Switzerland, and 1995 he worked on holography in Orsay-Paris, France, at the Institute for Theoretical and Applied Optics (IOTA).

Hans Peter Herzig: CV already submitted as Editor of this special issue.
Omar Manzardo received the diploma in physics from the University of Neuchâtel, Switzerland in 1994. In 1995, he was a scientific collaborator with the Biomedical Engineering Laboratory of the Swiss Federal Institute of Technology in Lausanne, Switzerland. In 1996, he joined the Applied Optics group at the Institute of Microtechnology of the University of Neuchâtel, Switzerland, as a PhD student and teaching assistant. His current research interests are micro-opto-electromechanical systems (MOEMS), and particularly microsized Fourier transform spectrometers.

Cornel Marxer was born on October 7, 1969, in Grabs, Switzerland. He received His M.Sc degree in 1994 from the Swiss Federal Institute of Technology in Lausanne and his $\mathrm{PhD}$ degree in 1997 from the University of Neuchâtel, Switzerland. In 1999 he was a founder of Sercalo Microtechnology Ltd. and acts as its CEO. His research activities are focused on silicon micromechanics for applications in fiber optic communications.

Kenneth J. Weible achieved his B.S.E.E. at the University of Michigan (Ann Arbor), then his M.S. in Optical Sciences at the University of Arizona and his $\mathrm{PhD}$ in Applied Optics at the University of Neuchâtel, Switzerland. Since 1996 he has been self-employed in the design and fabrication of micro-optical elements. Interests include: microlens arrays and diffractive optical elements.

René Dändliker: CV already submitted for paper no. 1019.

Nicolaas F. de Rooij joined the Institute of Microtechnology of the University of Neuchâtel, Switzerland (IMT UNI-NE), as professor and head of the Sensors, Actuators and Microsystems Laboratory in 1982. Since October 1990 till October 1996, he was acting as director of the IMT UNI-NE. He lectured at the Swiss Federal Institute of Technology, Zurich (ETHZ), and since 1989, he has been a part-time professor at the Swiss Federal Institute of Technology, Lausanne (EPFL). His research activities include microfabricated sensors, actuators, and microsystems. He was a member of the steering committee of the International Conference on Solid-State Sensors and Actuators and of Eurosensors. He acted as European Program Chairman of Transducers '87 and General Chairman of Transducers ' 89 . He is a member of the editorial boards for the journals Sensors and Actuators, Sensors and Materials and the IEEE Journal of Microelectromechanical Systems. 